\title{
RAS mutation: site of disease and recurrence pattern in colorectal cancer
}

\author{
Pierre E. Bonnot ${ }^{1,2}$, Guillaume Passot ${ }^{1,2}$ \\ ${ }^{1}$ Department of Surgical Oncology, CHU Lyon Sud, Hospices Civils de Lyon, Pierre-Bénite, France; ${ }^{2}$ Department of Surgical Oncology, University \\ of Lyon 1, Lyon, France \\ Contributions: (I) Conception and design: All authors; (II) Administrative support: All authors; (III) Provision of study materials or patients: All \\ authors; (IV) Collection and assembly of data: All authors; (V) Data analysis and interpretation: All authors; (VI) Manuscript writing: All authors; (VII) \\ Final approval of manuscript: All authors. \\ Correspondence to: Guillaume Passot. Department of Surgical Oncology, CHU Lyon Sud, Hospices Civils de Lyon, 165 Chemin du Grand Revoyet, \\ 69310 Pierre-Bénite, France. Email: guillaume.passot@chu-lyon.fr.
}

\begin{abstract}
Somatic mutation status in metastatic colorectal cancer (CRC), and namely mutational activation of the Kirsten rat sarcoma viral oncogene homolog (KRAS) oncogene, is becoming more and more relevant in clinical practice. In this review, we describe the current data about the importance of associations between the mutational activation status of the KRAS oncogene and clinical outcomes, prognosis and metastatic patterns of CRC. The presence of a KRAS mutation is detected in approximately $30-50 \%$ of CRC and represents a powerful predictor of oncologic outcomes. It is associated with low response to systemic chemotherapy and for insensitivity to the anti-EGFR antibodies in the preoperative setting. It is more frequently associated with right colon cancer. In non-metastatic patients, KRAS mutation leads to more aggressive disease with shorter recurrence free survival (RFS) and more lung recurrences. After resection of CRC liver metastases (LiM), KRAS mutation is directly associated with increased risk of recurrence, worse overall survival (OS), and a distinct metastatic pattern with more invasive intrahepatic recurrence and increased recurrence outside of the liver, particularly in the lung, the peritoneum, and even in uncommon metastatic sites such as the brain and bones. As metastasectomy with curative intent is increasingly considered, a comprehensive approach of tumor biology is required to face the specific challenge of patients with metastatic CRCs. Thus, as it represents one of the strongest predictors of oncologic outcomes, integrating the KRAS mutational status at all the different stages of patient care appears crucial in order to adapt both medical and surgical strategies.
\end{abstract}

Keywords: Kirsten rat sarcoma viral oncogene homolog (KRAS); colorectal cancer (CRC); metastases; chemotherapy

Submitted Aug 02, 2019. Accepted for publication Aug 12, 2019.

doi: $10.21037 /$ cco.2019.08.11

View this article at: http://dx.doi.org/10.21037/cco.2019.08.11

\section{Introduction}

Colorectal cancer (CRC) represents the third leading cause of cancer-related death worldwide. Approximately $15 \%$ to $25 \%$ of patients present with synchronous metastasis at diagnosis and $25 \%$ to $50 \%$ will develop metachronous distant disease and distant recurrence often occurs within 3 years. The most common site of CRC metastases is the liver, followed by lungs and peritoneum. This recurrence pattern will directly guide the optimal surveillance strategy after curative treatment of CRC, namely in the metastatic setting. Advances in cytotoxic agents and targeted therapies have led to major control of metastatic disease, allowing curative intent surgery and resulting in real improvement in overall survival (OS) for patients with metastatic CRC (1).

Carcinogenesis of CRC is directly linked to activating mutations in oncogenes. In CRC, different signal pathways can be affected such as the mitogen-activated kinase (MAPK) pathway or the phosphatidylinositol 3-kinase (PI3K) pathway. Among the MAPK pathway genes, Kirsten 
rat sarcoma viral oncogene homolog (KRAS) mutations are the most prevalent predictive and prognostic activated oncogene, detected in approximately $30-50 \%$ of CRCs. Currently, evaluation for mutations in KRAS in metastatic CRC is part of standard of care in order to select patients to treatment targeting the epidermal growth factor receptor (EGFR), as the presence of a KRAS mutation predicts for insensitivity to the anti-EGFR antibodies $(2,3)$. Furthermore, it is now well established that it is associated with poor recurrence free (RFS) and OS after liver resection, making RAS mutation status as a powerful predictor of oncologic outcomes $(4,5)$. In addition, the somatic mutation profile may also predict the pattern of metastatic spread in metastatic CRC and future prognosis. Metastasectomy with curative intent becomes increasingly considered and possible in metastatic CRC (6-8). Understanding and considering tumor biology as a powerful predictor of outcome may seem crucial in a modern and tailored approach to guide patient selection, treatment and surveillance in both pre and post therapeutic workups for CRC patients $(1,9,10)$.

Therefore, in this review, we describe the current data about the importance of associations between the mutational activation status of the KRAS oncogene and clinical outcomes, disease prognosis and metastatic patterns of CRC.

\section{Impact of RAS mutation in non-metastatic CRC}

In nonmetastatic CRC patients, prevalence of KRAS mutation ranges from $30 \%$ to $38 \%$. Patients with mutated KRAS seem more associated with right colon cancer than left colon cancer $(60 \%$ vs. $40 \%$, OR $2.05, \mathrm{P}<0.001)$, low grade tumor $(\mathrm{OR} 0.73, \mathrm{P}=0.007)$ and have less deficient mismatch repair status (OR $0.21, \mathrm{P}<0.001)$. They are also less likely to have a first degree relative with CRC or to be a smoker (11). Similar results concerning the association between KRAS mutation and right colon cancer were reported $(12,13)$.

\section{Oncologic outcomes associated with RAS mutation in non- metastatic patients}

Molecular testing and genotyping do not represent standard for non-metastatic patients $(14,15)$. The prognostic impact of RAS mutation in this population is inconsistent $(2,16,17)$. Stage I CRC has excellent prognosis with 5 -year OS up to 95\%. However, Reggiani Bonetti et al. (18) evaluated the impact of RAS mutational status in 62 stage I CRC. A RAS mutation was present in $40 \%$ of patients and was statistically associated with worse 5 -years OS $(\mathrm{P}=0.019)$. Yoon et al. studied in a large cohort of 2,478 patients with resected stage III CRC receiving adjuvant FOLFOX alone or combined with cetuximab the impact of RAS mutation. A RAS mutation in codon 12 was present in $31 \%$ of patients and $8.9 \%$ had mutation in codon 13 . KRAS mutations in either codon 12 (HR 1.52, $\mathrm{P}<0.0001$ ) or 13 (HR 1.36, $\mathrm{P}=0.0248$ ) were associated with shorter DFS (19). Similar results were obtained in another large cohort of 2,720 patients with $35 \%$ having a RAS mutation. Wild-type patients had 70.7\% 5 -year DFS versus $61 \%$ in mutated patients (16). It seems also that mutational status appears as a predictive biomarker of the benefit of adjuvant chemotherapy. Indeed, Deng et al. observed no benefit in 3-year DFS of an adjuvant FOLFOX in stage III CRC among patients showing wild-type RAS (84.3\% vs. $82.0 \%, \mathrm{P}=0.661$ ), whereas adjuvant treatment significantly improved outcomes in patients with RAS tumors (74.4.0\% vs. $50.2 \%, \mathrm{P}=0.020)(20)$.

Finally, KRAS mutation not only impacts time of recurrence but also its patterns. Tie et al. showed that RAS mutation in stages II and III CRC was associated with more lung recurrences (HR 2.1, $\mathrm{P}=0.007)$. However, it was not associated with liver relapse (21).

Thus, it seems that tumor biology, namely RAS mutation profile, provides important prognostic information as well in non-metastatic CRC that could guide adjuvant treatments or surveillance modalities and therefore needs further investigations.

\section{Impact of RAS mutation in metastatic CRC}

\section{Epidemiology of RAS mutations in metastatic CRC}

Prevalence of KRAS mutation among metastatic CRC patients range from $25 \%$ to $52 \%$ (22).

Concordance between primary tumor and metastases concerning overall oncogene mutational status is high ranging between $85 \%$ to $100 \%$, irrespective of patient medical history or treatment duration, highlighting that evaluation of the primary tumor appears as a sufficient and reasonable surrogate to establish the therapeutic strategy in the metastatic setting (23-27).

At diagnosis of metastatic disease, RAS mutant and wild-type tumors differed in the pattern of metastatic involvement. Among a cohort of 918 patients from Memorial Sloan Kettering Cancer Center, RAS mutant 
patients exhibited a higher incidence of lung metastasis at diagnosis compared to the wild-type cases. Liver, lung, bone, and brain involvement at the time of diagnosis of metastatic disease occurred in $75 \%, 13 \%, 0.6 \%$, and $0.2 \%$ of $R A S$ wildtype, respectively, and in $74 \%, 22 \%, 0.9 \%$, and $0.5 \%$ of $R A S$ mutant patients, respectively. Metastases were limited to the liver at the time of diagnosis in 303 (64\%) RAS wild-type and 245 (56\%) RAS mutant cases ( $\mathrm{P}=0.015)$ (13).

\section{Impact of RAS mutation in liver metastases (LiM)}

Approximately, $15 \%$ to $25 \%$ of patients present with $\mathrm{LiM}$ at diagnosis, and another $25 \%$ to $50 \%$ will develop metachronous hepatic disease within 3 years. Among them, KRAS mutant represents $18 \%$ to $41 \%$ of patients (1). It is now well established that KRAS mutant metastatic CRC is associated with worse oncologic outcomes with a higher risk of recurrence and decrease OS following curative resection of $\operatorname{LiM}(4,5,10,22,28)$. From the experience of the MD Anderson Cancer Center, Vauthey et al. found that patients with KRAS mutant metastatic CRC experienced worse RFS (HR 1.9, $\mathrm{P}=0.005)$ and $\mathrm{OS}(\mathrm{HR} 2.3, \mathrm{P}=0.002)$ compared with KRAS wild-type patients who underwent single-regimen modern chemotherapy before resection of LiM. The 3-year RFS and OS after curative resection were $33.5 \%$ and $81 \%$ for $R A S$ wild-type patients and $13.5 \%$ and $52.2 \%$ for $R A S$ mutant $(\mathrm{P}=0.001$ and $\mathrm{P}=0.002)$ (4). In a cohort of 169 patients from Memorial Sloan Kettering Cancer Center, Kemeny et al. found similar results with 3 -year RFS and OS of $30 \%$ and $81 \%$ in KRAS mutated patients versus $46 \%$ and $95 \%$ in wild-type patients with resected LiM and adjuvant hepatic arterial infusion plus systemic therapy (5). Presence of KRAS mutations does not only affect the time of recurrence after resection of LiM, it also determines its patterns. Indeed, in both cohorts, RAS mutation was associated with an increased incidence of recurrence in lung, bone and brain in comparison to wildtype patients $(4,5)$. Yaeger et al. confirmed that RAS mutant was predictive of involvement of these sites (HR 1.5, 1.6 and 3.7, respectively). Cumulative incidences at 2 years were $32.5 \%$ versus $19 \%$ for lung ( $\mathrm{P}=0.001), 8.8 \%$ versus $4.4 \%$ for bone $(\mathrm{P}=0.024)$, and $1.4 \%$ versus $0.2 \%$ for brain metastasis $(\mathrm{P}<0.01)(13)$. However, the cumulative incidence of liver metastasis did not vary by $R A S$ mutation status ( $12 \%$ versus $14.3 \%, \mathrm{P}=0.78)$. RAS mutation appears also associated with more aggressive recurrences not amenable to second or salvage curative treatments. Okuno et al. studied 566 patients with recurrence after hepatectomy for LiM, of whom 309 (54.6\%) underwent chemotherapy only, 189 (33.4\%) surgical resection, 47 (8.3\%) ablation and 21 (3.7\%) radiation therapy. Local therapy was statistically associated with major improved OS compare to chemotherapy only, and RAS mutation was associated with recurrence not amenable to local therapy (HR $1.49, \mathrm{P}=0.001$ ). It was also associated with worse survival in both patients who received local therapy and those who received chemotherapy only (29).

Secondly, several clinical scores and nomograms mainly based on clinicopathological factors were created in order to guide selection of patients who would benefit from resection of LiM (30-33). As RAS mutation status may represent a direct measure of tumor biology and appears strongly linked to oncologic outcomes, it has been recently included in a new and modified predictive score in order to offer a better potential guide for patients selection. Indeed, replacing traditional clinicopathological factors such as disease free survival, number of metastases and CEA level with RAS mutation in addition to lymph node positive primary status and size of metastases in the Memorial Sloan Kettering Clinical Score (traditional clinical score, $\mathrm{t}-\mathrm{CS}$ ) resulted in an modified clinical score (m-CS) that outperformed the $\mathrm{t}-\mathrm{CS}$, and thus providing a quick and easy preoperative assessment of the expected survival benefit (10). Similarly, especially for patients with RAS mutated LiM, Passot et al. proposed a predictive model defining and combining three risk factors-node-positive primary tumor, largest LiM $>3 \mathrm{~cm}$ and $>7$ cycles of preoperative chemotherapyin order to help selection of candidates to hepatectomy. High-risk patients with the 3 risk factors demonstrated poorer median OS of 21.5 months versus 57 and 41 months for patients with 1 or 2 risk factors, respectively. Thus, alternative therapies or further systemic therapy would be preferable for those patients (34). Finally, as next-generation sequencing technology becomes widely available, multigene analysis is possible and could provide a more accurate risk stratification based directly on tumor biology. For instance, a concomitant RAS and TP53 mutation is associated with decreased survival after resection than RAS mutation only, and patients with a high evolutionary action score TP53 mutation showed even a worse prognosis (9). Those results underline that it is time to change the paradigm of traditional poor prognostic factors, such as multiple LiM or extrahepatic disease. Such patients could be considered for surgery if their mutational status is favorable. Further investigations using high-throughput genomic analysis are needed to validate those results and to optimize patient selection for surgery. 
Finally, tumor biology could be also directly integrated in the surgical strategy. Indeed, non-anatomical resections and parenchymal sparring represent standard of care for patients with LiM. In the era of modern chemotherapy, it appears that a $1 \mathrm{~mm}$ resection margin could be sufficient to obtain acceptable oncologic outcomes (35-37). Furthermore, it seems that a positive resection margin does not worsen survival in patients with a major pathologic response to preoperative chemotherapy (38). However, RAS mutations are associated with less optimal radiologic morphologic response and major pathologic response after modern chemotherapy that included bevacizumab before resection of LiM (39). In a study including 633 patients who underwent surgery for LiM, of whom 229 had mutant RAS, RAS mutation was significantly associated with positive resection margin rate $11.4 \%$ versus $5.4 \%$ for wildtype RAS patients $(\mathrm{P}=0.007)$, suggesting a more aggressive intrahepatic growth pattern. A positive margin (HR 3.36) and RAS mutation (HR 1.629) were independently associated with worse OS (40). Similarly, presence of RAS mutation is associated for more diaphragmatic invasion by liver metastasis requiring major hepatic resection and diaphragmatic resection (41). Margonis et al. suggested that anatomical resections with more extensive surgical margins could counterbalance this adverse and more invasive genetic profile associated to RAS mutation (42). Another concept for limiting systemic toxicity while intensifying treatment in the adjuvant setting for such patients is hepatic arterial infusion $(5,43)$. However, these different strategies for controlling hepatic recurrence in RAS mutated patients with $\mathrm{LiM}$ need further investigations in randomized trials.

\section{Impact of RAS mutation in lung metastases (LuM)}

LuM will eventually develop in $5-15 \%$ of patients with $\mathrm{mCRC}$ and prognosis after metastasectomy ranges between $41 \%$ and $56 \%$ (44). The presence of a KRAS mutation appears directly associated to LuM. In non-metastatic patients with resected stage II or III CRC, RAS mutated patients experienced more lung recurrence (HR 2.1, $\mathrm{P}=0.007$ ) (21). In a large Australian cohort of 5,967 patients with mCRC, RAS mutation was significantly associated with lung-only metastases (HR 1.4, $\mathrm{P}=007$ ) (45).

Secondly, in mCRC, KRAS mutation influences directly oncologic outcomes. In patients with resected LiM, RAS mutation is associated with worse lung RFS $(4,5,13)$. Presence of a KRAS mutation represents also a strong predictive factor of poor OS in case of LuM. Ghidini et al. studied lung specimens from 75 mCRC among whom $36 \%$ had KRAS mutation. Median OS was 60.9 for wild-type patients versus 36.6 months in mutated patients $(\mathrm{P}=0.035)$. In multivariate analysis, presence of a KRAS mutation was statistically associated with worse OS (HR 2.17, $\mathrm{P}=0.012$ ) (46). Similar results were obtained by Renaud et al. in a cohort of 180 patients with lung metastasectomy. Molecular analysis revealed mutated KRAS in 93 patients $(51.7 \%)$ and mutated BRAF in 19 patients (10.6\%). The 5 -year OS was $0 \%$ for mutated BRAF, $44 \%$ for mutated KRAS and $100 \%$ for wild-type, with corresponding median OS of 15, 55 and 98 months, respectively ( $\mathrm{P}=0.001)$ (47). In mutated patients, it seems also that according to KRAS amino-acid substitution, different signaling pathways are activated, resulting in different tumor evolution and clinical outcomes. After lung metastasectomy, KRAS exon 2 codon 13 mutation is associated with, better OS (82 vs. 54 months, $\mathrm{P}=0.009$ ) and lung RFS (78 versus 56 months, $\mathrm{P}=0.008$ ) than codon 12 (48). Similarly to LiM, Renaud et al. showed also that anatomical resection with segmentectomy compared to wedge resections of LuM could improve OS in RAS mutated patients. However, in wild-type patients, the type of resection did not impact OS (8).

\section{Impact of RAS mutation in peritoneal metastases (PM)}

In CRC, up to $15 \%$ of patients will develop PM that carry a worse prognosis than other metastases (49). However, since a few decades, development of cytoreductive surgery (CRS) with or without peroperative hyperthermic intraperitoneal chemotherapy (HIPEC) leads to prolonged survival with median survival ranging from 30 to 45 months in comparison to dismal prognosis associated to systematic treatment only $(50,51)$. Survival is undoubtedly linked to the crucial surgical endpoint: completeness of CRS, which is directly associated to the extent and distribution of PM $(7,52)$. However, the role of tumor biology and RAS mutations in the context of PM remained unclear until recent studies (53-57). RAS mutation is associated with peritoneal recurrence after resection of LiM (41) and prevalence of RAS mutations appears higher in PM than in LiM, up to $58 \%$ (55). In 524 patients who underwent CRS and HIPEC for CRC PM, 378 had known RAS/ RAF status. Among them, 186 (49.2\%) had a RAS/RAF mutation. KRAS (HR 1.46) and BRAF (HR 3.97) were both associated with impaired OS and also shorter RFS. Based on those results, a point-based risk score termed BIOSCOPE, including RAS/RAF mutational status, PCI, and $\mathrm{N}$ - and G-status of the primary tumor was developed to determine 4 risk groups with distinct prognosis in order to 
guide patient selection (56). Similarly, Arjona-Sanchez et al. reported a significant decrease in OS for patients with RAS mutated tumors (HR 2.024, $\mathrm{P}=0.045$ ). Thus, they associated RAS mutational status to PSDSS, the most widely used and validated score to select patient for CRS and HIPEC, and created a new score, RAS-PSDSS, that also outperformed the former PSDSS (57).

\section{Impact of $R A S$ mutation in bone metastases (BoM)}

BoM in patients with CRC are relatively uncommon and incidence occurs in 3\% to $7 \%$. Median OS after diagnosis of BoM ranged from 5 to 21 months. Risk factors usually described are rectal cancer, primary lymph node invasion and lung metastases (58). KRAS status appears also to be predictive of BoM. Kemeny et al. found a cumulative recurrence for BoM by 3 years of $13.4 \%$ in KRAS mutated versus $2 \%$ in KRAS wild patients (5). At 2 years, Yaeger et al. showed similar results with a cumulative incidence of BoM of $8.8 \%$ with RAS mutated tumors versus $4.4 \%$, in wild-type patients $(\mathrm{P}=0.024)$. RAS mutation remained an independent predictor of BoM in multivariate analysis (HR 1.62, $\mathrm{P}=0.012$ ) (13).

\section{Impact of RAS mutation in brain metastases (BM)}

$\mathrm{BM}$ represent an uncommon metastatic site in CRC. However, consequences for affected patients are major. They are reported to develop late in the disease and patients normally have metastases to other organs at diagnosis, namely in the lung (59). Incidence of BM ranges from $0.6 \%$ to $3.2 \%$ (60). However, in selected patients, resection or radioablation of oligometastatic disease may provide benefits with median survival ranging from 2 to 9 months for non-resected patients versus 10 to 16 months for patients with loco-regional treatments (61). KRAS mutation is directly associated with higher prevalence of BM from CRC and several studies investigated this potential association (27,61-63). Tie et al. found that $56.5 \%$ of patients with BM had RAS mutations (21). Yaeger et al. showed that patients with RAS mutated tumors had higher incidence of $\mathrm{BM}$ (6.1\% versus $1.9 \%)$ than wild-type patients (HR 3.7, $\mathrm{P}<0.01$ ) (13). After resection of LiM, Kemeny et al. found a cumulative recurrence by 3 years of $14.5 \%$ in KRAS mutated versus $2 \%$ in KRAS wild patients (5). KRAS mutation was an independent predictor of spread to the brain in both studies. Thus, an understanding of this specific pattern of spread and recurrence may alert physicians to look for specific neurologic symptoms and to perform cerebral CT scans in $R A S$ mutant metastatic CRC.

\section{Conclusions}

In both non-metastatic and metastatic CRCs, RAS mutations not only predict strongly oncologic outcomes with less response to chemotherapy, worse RFS and OS, but also have direct impact on the site of disease and recurrence patterns. RAS mutated status is more frequently associated with right side colon cancer, and mutations in the primary tumor remain concordant with distant metastasis. It seems not directly associated with LiM but appears more frequent in LuM or PM and uncommon metastatic sites such as BoM and BM. Integration of RAS mutations in the selection of patients for curative intent surgery in metastatic CRC appears crucial and may render traditional clinicopathological risk factor obsolete. Further investigations using namely high-throughput genomic analysis are needed to validate this new paradigm.

\section{Acknowledgments}

None.

\section{Footnote}

Conflicts of Interest: The authors have no conflicts of interest to declare.

Ethical Statement: The authors are accountable for all aspects of the work in ensuring that questions related to the accuracy or integrity of any part of the work are appropriately investigated and resolved.

\section{References}

1. Van Cutsem E, Cervantes A, Nordlinger B, et al. Metastatic colorectal cancer: ESMO Clinical Practice Guidelines for diagnosis, treatment and follow-up. Ann Oncol 2014;25:iii1-9.

2. De Roock $W$, De Vriendt V, Normanno N, et al. KRAS, BRAF, PIK3CA, and PTEN mutations: implications for targeted therapies in metastatic colorectal cancer. Lancet Oncol 2011;12:594-603.

3. Allegra CJ, Rumble RB, Hamilton SR, et al. Extended RAS Gene Mutation Testing in Metastatic Colorectal Carcinoma to Predict Response to Anti-Epidermal Growth 
Factor Receptor Monoclonal Antibody Therapy: American Society of Clinical Oncology Provisional Clinical Opinion Update 2015. J Clin Oncol 2016;34:179-85.

4. Vauthey JN, Zimmitti G, Kopetz SE, et al. RAS Mutation Status Predicts Survival and Patterns of Recurrence in Patients Undergoing Hepatectomy for Colorectal Liver Metastases: Ann Surg 2013;258:619-26; discussion 626-7.

5. Kemeny NE, Chou JF, Capanu M, et al. KRAS mutation influences recurrence patterns in patients undergoing hepatic resection of colorectal metastases: KRAS Mutation Influences Recurrences. Cancer 2014;120:3965-71.

6. Adam R, Wicherts DA, de Haas RJ, et al. Patients With Initially Unresectable Colorectal Liver Metastases: Is There a Possibility of Cure? J Clin Oncol 2009;27:1829-35.

7. Goéré D, Malka D, Tzanis D, et al. Is There a Possibility of a Cure in Patients With Colorectal Peritoneal Carcinomatosis Amenable to Complete Cytoreductive Surgery and Intraperitoneal Chemotherapy? Ann Surg 2013;257:1065-71.

8. Renaud S, Seitlinger J, Lawati YA, et al. Anatomical Resections Improve Survival Following Lung Metastasectomy of Colorectal Cancer Harboring KRAS Mutations. Ann Surg 2018. [Epub ahead of print].

9. Chun YS, Passot G, Yamashita S, et al. Deleterious Effect of RAS and Evolutionary High-risk TP53 Double Mutation in Colorectal Liver Metastases. Ann Surg 2019;269:917-23.

10. Brudvik KW, Jones RP, Giuliante F, et al. RAS Mutation Clinical Risk Score to Predict Survival After Resection of Colorectal Liver Metastases. Ann Surg 2019;269:120-6.

11. Gonsalves WI, Mahoney MR, Sargent DJ, et al. Patient and Tumor Characteristics and BRAF and KRAS Mutations in Colon Cancer, NCCTG/Alliance N0147 [Internet]. JNCI J Natl Cancer Inst 106, 2014 [cited 2019 Jul 23]. Available online: https://academic.oup.com/jnci/ article-lookup/doi/10.1093/jnci/dju106

12. Tong JH, Lung RW, Sin FM, et al. Characterization of rare transforming KRAS mutations in sporadic colorectal cancer. Cancer Biol Ther 2014;15:768-76.

13. Yaeger R, Cowell E, Chou JF, et al. RAS mutations affect pattern of metastatic spread and increase propensity for brain metastasis in colorectal cancer: RAS Mutation Impact on CRC Metastasis. Cancer 2015;121:1195-203.

14. Labianca R, Nordlinger B, Beretta GD, et al. Early colon cancer: ESMO Clinical Practice Guidelines for diagnosis, treatment and follow-up. Ann Oncol 2013;24:vi64-72.

15. Schmoll HJ, Van Cutsem E, Stein A, et al. ESMO Consensus Guidelines for management of patients with colon and rectal cancer. A personalized approach to clinical decision making. Ann Oncol 2012;23:2479-516.

16. Sinicrope FA, Shi Q, Smyrk TC, et al. Molecular Markers Identify Subtypes of Stage III Colon Cancer Associated With Patient Outcomes. Gastroenterology 2015;148:88-99.

17. Sinicrope FA, Shi Q, Allegra CJ, et al. Association of DNA Mismatch Repair and Mutations in BRAF and KRAS With Survival After Recurrence in Stage III Colon Cancers: A Secondary Analysis of 2 Randomized Clinical Trials. JAMA Oncol 2017;3:472.

18. Reggiani Bonetti L, Barresi V, Maiorana A, et al. Clinical Impact and Prognostic Role of KRAS/BRAF/PIK3CA Mutations in Stage I Colorectal Cancer. Dis Markers 2018;2018:2959801.

19. Yoon HH, Tougeron D, Shi Q, et al. KRAS Codon 12 and 13 Mutations in Relation to Disease-Free Survival in BRAF-Wild-Type Stage III Colon Cancers from an Adjuvant Chemotherapy Trial (N0147 Alliance). Clin Cancer Res 2014;20:3033-43.

20. Deng Y, Wang L, Tan S, et al. KRAS as a predictor of poor prognosis and benefit from postoperative FOLFOX chemotherapy in patients with stage II and III colorectal cancer. Mol Oncol 2015;9:1341-7.

21. Tie J, Lipton L, Desai J, et al. KRAS Mutation Is Associated with Lung Metastasis in Patients with Curatively Resected Colorectal Cancer. Clin Cancer Res 2011;17:1122-30.

22. Tsilimigras DI, Ntanasis-Stathopoulos I, Bagante F, et al. Clinical significance and prognostic relevance of KRAS, BRAF, PI3K and TP53 genetic mutation analysis for resectable and unresectable colorectal liver metastases: A systematic review of the current evidence. Surg Oncol 2018;27:280-8.

23. Oudejans JJ, Slebos RJ, Zoetmulder FA, et al. Differential activation of ras genes by point mutation in human colon cancer with metastases to either lung or liver. Int J Cancer 1991;49:875-9.

24. Santini D, Loupakis F, Vincenzi B, et al. High Concordance of KRAS Status Between Primary Colorectal Tumors and Related Metastatic Sites: Implications for Clinical Practice. The Oncologist 2008;13:1270-5.

25. Italiano A, Hostein I, Soubeyran I, et al. KRAS and BRAF Mutational Status in Primary Colorectal Tumors and Related Metastatic Sites: Biological and Clinical Implications. Ann Surg Oncol 2010;17:1429-34.

26. Knijn N, Mekenkamp LJ, Klomp M, et al. KRAS mutation analysis: a comparison between primary tumours and matched liver metastases in 305 colorectal cancer patients. Br J Cancer 2011;104:1020-6. 
27. Kawamoto Y, Tsuchihara K, Yoshino T, et al. KRAS mutations in primary tumours and post-FOLFOX metastatic lesions in cases of colorectal cancer. Br J Cancer 2012;107:340-4.

28. Stremitzer S, Stift J, Gruenberger B, et al. KRAS status and outcome of liver resection after neoadjuvant chemotherapy including bevacizumab. Br J Surg 2012;99:1575-82.

29. Okuno M, Goumard C, Kopetz S, et al. RAS Mutation is Associated with Unsalvageable Recurrence Following Hepatectomy for Colorectal Cancer Liver Metastases. Ann Surg Oncol 2018;25:2457-66.

30. Fong Y, Fortner J, Sun RL, et al. Clinical score for predicting recurrence after hepatic resection for metastatic colorectal cancer: analysis of 1001 consecutive cases. Ann Surg 1999;230:309.

31. Rees M, Tekkis PP, Welsh FK, et al. Evaluation of Longterm Survival After Hepatic Resection for Metastatic Colorectal Cancer: A Multifactorial Model of 929 Patients. Ann Surg 2008;247:125-35.

32. Kattan MW, Gönen M, Jarnagin WR, et al. A Nomogram for Predicting Disease-specific Survival After Hepatic Resection for Metastatic Colorectal Cancer. Ann Surg 2008;247:282-7.

33. Zakaria S, Donohue JH, Que FG, et al. Hepatic Resection for Colorectal Metastases: Value for Risk Scoring Systems? Ann Surg 2007;246:183-91.

34. Passot G, Denbo JW, Yamashita S, et al. Is hepatectomy justified for patients with RAS mutant colorectal liver metastases? An analysis of 524 patients undergoing curative liver resection. Surgery 2017;161:332-40.

35. de Haas RJ, Wicherts DA, Flores E, et al. R1 Resection by Necessity for Colorectal Liver Metastases: Is It Still a Contraindication to Surgery? Ann Surg 2008;248:626-37.

36. Ayez N, Lalmahomed ZS, Eggermont AM, et al. Outcome of Microscopic Incomplete Resection (R1) of Colorectal Liver Metastases in the Era of Neoadjuvant Chemotherapy. Ann Surg Oncol 2012;19:1618-27.

37. Laurent C, Adam JP, Denost Q, et al. Significance of R1 Resection for Advanced Colorectal Liver Metastases in the Era of Modern Effective Chemotherapy. World J Surg 2016;40:1191-9.

38. Andreou A, Aloia TA, Brouquet A, et al. Margin Status Remains an Important Determinant of Survival After Surgical Resection of Colorectal Liver Metastases in the Era of Modern Chemotherapy. Ann Surg 2013;257:1079-88.

39. Zimmitti G, Shindoh J, Mise Y, et al. RAS Mutations Predict Radiologic and Pathologic Response in Patients Treated with Chemotherapy Before Resection of Colorectal
Liver Metastases. Ann Surg Oncol 2015;22:834-42.

40. Brudvik KW, Mise Y, Chung MH, et al. RAS Mutation Predicts Positive Resection Margins and Narrower Resection Margins in Patients Undergoing Resection of Colorectal Liver Metastases. Ann Surg Oncol 2016;23:2635-43.

41. Okuno M, Gourmard C, Mizuno T, et al. Pathological diaphragmatic invasion by colorectal liver metastases is associated with RAS mutation, peritoneal recurrence and worse survival. HPB 2018;20:57-63.

42. Margonis GA, Buettner S, Andreatos N, et al. Anatomical Resections Improve Disease-free Survival in Patients With KRAS-mutated Colorectal Liver Metastases. Ann Surg 2017;266:641-9.

43. Groot Koerkamp B, Sadot E, Kemeny NE, et al. Perioperative hepatic arterial infusion pump chemotherapy is associated with longer survival after resection of colorectal liver metastases: A propensity score analysis. J Clin Oncol 2017;35:1938-44.

44. Kim HK. Pulmonary metastasectomy for colorectal cancer: How many nodules, how many times? World J Gastroenterol 2014;20:6133.

45. Prasanna T, Karapetis CS, Roder D, et al. The survival outcome of patients with metastatic colorectal cancer based on the site of metastases and the impact of molecular markers and site of primary cancer on metastatic pattern. Acta Oncol 2018;57:1438-44.

46. Ghidini M, Personeni N, Bozzarelli S, et al. KRAS mutation in lung metastases from colorectal cancer: prognostic implications. Cancer Med 2016;5:256-64.

47. Renaud S, Romain B, Falcoz PE, et al. KRAS and BRAF mutations are prognostic biomarkers in patients undergoing lung metastasectomy of colorectal cancer. Br J Cancer 2015;112:720-8.

48. Renaud S, Guerrera F, Seitlinger J, et al. KRAS exon 2 codon 13 mutation is associated with a better prognosis than codon 12 mutation following lung metastasectomy in colorectal cancer. Oncotarget 2017;8:2514-24.

49. Franko J, Shi Q, Meyers JP, et al. Prognosis of patients with peritoneal metastatic colorectal cancer given systemic therapy: an analysis of individual patient data from prospective randomised trials from the Analysis and Research in Cancers of the Digestive System (ARCAD) database. Lancet Oncol 2016;17:1709-19.

50. Quenet F, Elias D, Roca L, et al. A UNICANCER phase III trial of hyperthermic intra-peritoneal chemotherapy (HIPEC) for colorectal peritoneal carcinomatosis (PC): PRODIGE 7. J Clin Oncol 2018;36. doi: 10.1200/ 
JCO.2018.36.18_suppl.LBA3503.

51. Verwaal VJ, van Ruth S, de Bree E, et al. Randomized Trial of Cytoreduction and Hyperthermic Intraperitoneal Chemotherapy Versus Systemic Chemotherapy and Palliative Surgery in Patients With Peritoneal Carcinomatosis of Colorectal Cancer. J Clin Oncol 2003;21:3737-43

52. Glehen O, Mohamed F, Gilly FN. Peritoneal carcinomatosis from digestive tract cancer: new management by cytoreductive surgery and intraperitoneal chemohyperthermia. Lancet Oncol 2004;5:219-28.

53. Gillern SM, Chua TC, Stojadinovic A, et al. KRAS Status in Patients With Colorectal Cancer Peritoneal Carcinomatosis and Its Impact on Outcome. Am J Clin Oncol 2010;33:456-60.

54. Massalou D, Benizri E, Chevallier A, et al. Peritoneal carcinomatosis of colorectal cancer: novel clinical and molecular outcomes. Am J Surg 2017;213:377-87.

55. Passot G, Kim BJ, Glehen O, et al. Impact of RAS Mutations in Metastatic Colorectal Cancer After Potentially Curative Resection: Does Site of Metastases Matter? Ann Surg Oncol 2018;25:179-87.

56. Schneider MA, Eden J, Pache B, et al. Mutations of RAS/ RAF Proto-oncogenes Impair Survival After Cytoreductive Surgery and HIPEC for Peritoneal Metastasis of Colorectal Origin. Ann Surg 2018;268:845-53.

57. Arjona-Sanchez A, Rodriguez-Ortiz L, Baratti D, et al. RAS Mutation Decreases Overall Survival After Optimal Cytoreductive Surgery and Hyperthermic Intraperitoneal

Cite this article as: Bonnot PE, Passot G. RAS mutation: site of disease and recurrence pattern in colorectal cancer. Chin Clin Oncol 2019;8(5):55. doi:10.21037/cco.2019.08.11
Chemotherapy of Colorectal Peritoneal Metastasis: A Modification Proposal of the Peritoneal Surface Disease Severity Score. Ann Surg Oncol 2019;26:2595-604.

58. Christensen TD, Jensen SG, Larsen FO, et al. Systematic review: Incidence, risk factors, survival and treatment of bone metastases from colorectal cancer. J Bone Oncol 2018;13:97-105.

59. Jung M, Ahn JB, Chang JH, et al. Brain metastases from colorectal carcinoma: prognostic factors and outcome. J Neurooncol 2011;101:49-55.

60. Christensen TD, Spindler KL, Palshof JA, et al. Systematic review: brain metastases from colorectal cancer-Incidence and patient characteristics [Internet]. BMC Cancer 16, 2016 [cited 2019 Jul 1]. Available online: http://bmccancer. biomedcentral.com/articles/10.1186/s12885-016-2290-5

61. Silva IL, Iskandarani M, Hotouras A, et al. A systematic review to assess the management of patients with cerebral metastases secondary to colorectal cancer. Tech Coloproctol 2017;21:847-52.

62. Tanriverdi O, Kaytan-Saglam E, Ulger S, et al. The clinical and pathological features of 133 colorectal cancer patients with brain metastasis: a multicenter retrospective analysis of the Gastrointestinal Tumors Working Committee of the Turkish Oncology Group (TOG) [Internet]. Med Oncol 31, 2014 [cited 2019 Jul 10]. Available online: http://link. springer.com/10.1007/s12032-014-0152-z

63. Magni E, Santoro L, Ravenda PS, et al. Brain metastases from colorectal cancer: main clinical factors conditioning outcome. Int J Colorectal Dis 2014;29:201-8. 